\title{
Saccadic Modulation of Neural Responses: Possible Roles in Saccadic Suppression, Enhancement, and Time Compression
}

\author{
Michael R. Ibbotson, ${ }^{1,2}$ Nathan A. Crowder, ${ }^{1}$ Shaun L. Cloherty, ${ }^{1}$ Nicholas S. C. Price, ${ }^{1,3}$ and Michael J. Mustari ${ }^{2}$ \\ ${ }^{1}$ Visual Sciences Group and Australian Research Council Centre of Excellence in Vision Science, School of Biology, Australian National University, \\ Canberra, Australian Capital Territory 2601, Australia, ${ }^{2}$ Visual Sciences, Yerkes National Primate Research Center, Emory University, Atlanta, Georgia \\ 30322, and ${ }^{3}$ Department of Neurobiology, Harvard Medical School, Boston, Massachusetts 02115-5701
}

Humans use saccadic eye movements to make frequent gaze changes, yet the associated full-field image motion is not perceived. The theory of saccadic suppression has been proposed to account for this phenomenon, but it is not clear whether suppression originates from a retinal signal at saccade onset or from the brain before saccade onset. Perceptually, visual sensitivity is reduced before saccades and enhanced afterward. Over the same time period, the perception of time is compressed and even inverted. We explore the origins and neural basis of these effects by recording from neurons in the dorsal medial superior temporal area (MSTd) of alert macaque monkeys. Neuronal responses to flashed presentations of a textured pattern presented at random times relative to saccades exhibit a stereotypical pattern of modulation. Response amplitudes are strongly suppressed for flashes presented up to 90 ms before saccades. Immediately after the suppression, there is a period of $200-450 \mathrm{~ms}$ in which flashes generate enhanced response amplitudes. Our results show that (1) MSTd is not directly suppressed, rather suppression is inherited from earlier visual areas; (2) early suppression of the visual system must be of extra-retinal origin; (3) postsaccadic enhancement of neural activity occurs in MSTd; and (4) the enhanced responses have reduced latencies. As a whole, these observations reveal response properties that could account for perceptual observations relating to presaccadic suppression, postsaccadic enhancement and time compression.

Key words: visual system; eye movements; saccadic suppression; postsaccadic enhancement; perception; macaque cortex

\section{Introduction}

The evolution of midget cell pathways in the primate fovea has provided the visual system with a small-field, high-acuity camera to view the world (Walls, 1963; Rodieck, 1988). However, by only having a small region of the retina devoted to high resolution vision it is necessary to move the eyes frequently to point the fovea at areas of interest, e.g., humans make 2-8 saccadic eye movements per second (for review, see Findlay and Gilchrist, 2003). Every saccade is associated with a transient but high speed displacement of the retinal image. Despite the frequency of saccades we never perceive these image displacements. A long standing theory suggests that visual sensitivity is reduced at the time of saccades to remove our perception of image motion (Holt, 1903; Ross et al., 2001). Perceptually, visual performance is indeed suppressed before and during saccades (Burr et al., 1982, 1994; Diamond et al., 2000). One hypothesis attributes this observation to a central mechanism that alerts the visual system of impending saccades (Duffy and Lombroso, 1968) (for review, see Ross et al., 2001). Such a warning signal must originate from saccade-related

Received Aug. 19, 2008; accepted Sept. 9, 2008.

This work was supported by the Australian Research Council Centre of Excellence in Vision Science (CE0561903) and National Institutes of Health Grants (EY06069, RR0165). We thank Drs. Markus Hietanen and Seiji Ono, and Tracey Broznya, Katia Peixoto, and Anthony Gazy for help with experiments and animal care.

Correspondence should be addressed to Dr. Michael R. Ibbotson, Visual Sciences Group, School of Biology, The Australian National University, GPO Box 475, Canberra, Australian Capital Territory 2601 Australia. E-mail: Michael.Ibbotson@anu.edu.au.

DOI:10.1523/JNEUROSCI.3950-08.2008

Copyright $\odot 2008$ Society for Neuroscience $\quad$ 0270-6474/08/2810952-09\$15.00/0 pathways in the brain, which are active before saccade onset (Sommer and Wurtz, 2002, 2006). An alternative hypothesis suggests that rapid acceleration of the eye at saccade onset causes physical distortion of the retina leading to a net decrement in retinal activity (Richards, 1969; Castet et al., 2001, 2002). It is suggested that, perceptually, this decrement is erroneously ascribed to presaccadic epochs because of the variable delays and long integration times inherent in the visual system (Ostendorf et al., 2006). Superficially, the observed presaccade reduction in visual perception (Diamond et al., 2000) is consistent with both hypotheses.

With the limitations of perceptual studies in mind, several researchers have looked for evidence of saccadic suppression at the neural level. Recordings from the lateral geniculate nucleus (LGN), the primary gateway to the visual cortex, show evidence for suppression of neural activity before saccades and enhancement afterward, although the effects are variable and complex (Lee and Malpeli, 1998; Ramcharan et al., 2001; Reppas et al., 2002; Royal et al., 2006). Studies in the parietal cortex [e.g., middle temporal (MT) and medial superior temporal areas (MSTd)] have also revealed suppression during saccades and postsaccadic enhancement (Thiele et al., 2002; Price et al., 2005; Ibbotson et al., 2007). However, the studies in parietal cortex have been limited to stimuli presented after saccade onset. Here we observe the responses of visual neurons in MSTd to stimuli delivered before and after saccade onset. In this way, we characterize the full time course of saccadic modulation of neural activity and show that 
T1 - weighted MRI
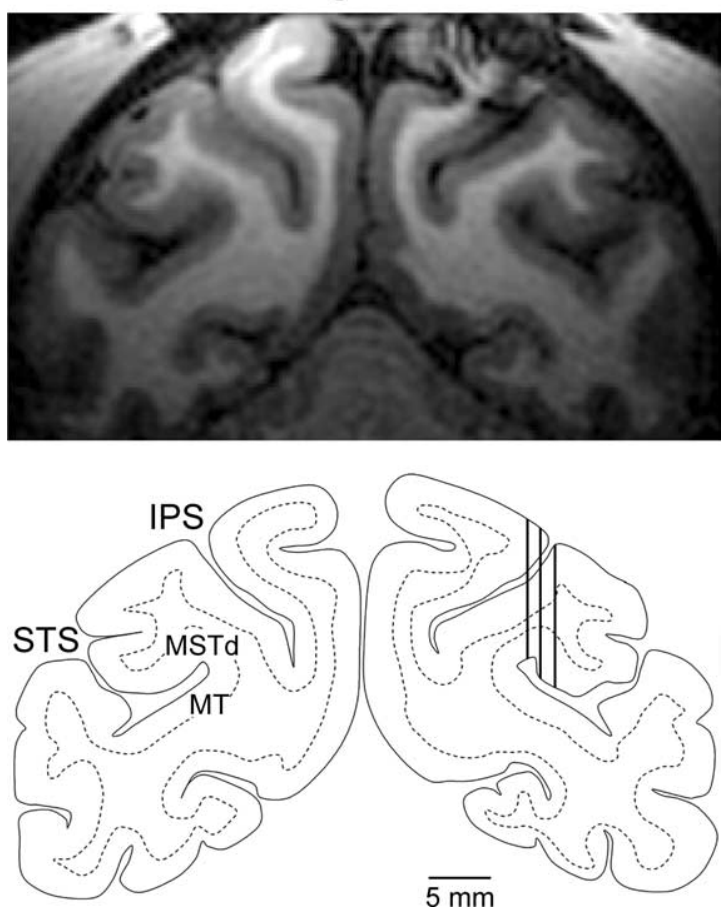

Figure 1. Recording site. Location of MST and medial-lateral range of electrode tracks in MSTd (top). Recoding sites of visual motion neurons in right MSTd verified by structural MRI (T1-weighted, fast spin-echo; Siemens; 3T magnet). Line drawing indicates representative recording tracks run in the coronal plane (bottom). Penetrations and unit depths were reconstructed by MRI and micro-drive readings taken from visual motion sensitive neurons. IPS, Intraparietal sulcus; STS, superior temporal sulcus. Some shadow artifacts from titanium bolts used to secure the recording chamber are evident on the upper right side. Scale bar, $5 \mathrm{~mm}$.

suppression most likely originates from an active mechanism before saccade onset. Furthermore, by comparing the spontaneous activity of MSTd neurons during saccades both in light and in darkness, we show that suppression does not occur in the dark but that postsaccadic enhancement does.

\section{Materials and Methods}

Surgical procedures. Data were collected from three juvenile rhesus monkeys (Macaca mulatta), one female and two male. All surgical and experimental procedures were performed in strict compliance with National Institutes of Health guidelines and protocols approved by the Institutional Animal Care and Use Committee at Emory University. Sterile surgical procedures were performed under aseptic conditions using isoflurane anesthesia (1.25-2.0\%) to stereotaxically implant an MRIcompatible head stabilization system (Crist Instruments) and a recording chamber centered above the superior temporal sulcus (lateral, 15 $\mathrm{mm}$; posterior, $5 \mathrm{~mm}$ ). A scleral search coil for measuring eye movements was implanted underneath the conjunctiva of both eyes.

Locations of recording sites were confirmed using a Siemens 3-Tesla magnetic resonance imager (MRI) located at the Yerkes National Primate Research Center. Imaging sessions to acquire 3-dimenesional T-1weighted images were performed under sedation (ketamine/telezol) and surgical levels of isoflurane (1.0-1.5\%). Vital signs including blood pressure, heart rate, body temperature, expired $\mathrm{CO}_{2}$ and blood oxygenation were continuously monitored and maintained at physiological levels. Monkeys were held in an MRI compatible stereotaxic frame (Crist Instruments) during imaging studies. We set the scan parameters to sample $1 \mathrm{~mm}$ slices through the entire anterior-posterior extent of the brain including the recording chambers mounted over MSTd (Fig. 1, top). We used Neurolens software to identify regions of interest below our Cilux recording chambers (Crist Instruments). We used an adjustable radiusand-angle positioning device that attached to the recording chamber to precisely place guide tubes and electrodes into MSTd. This device includes a centering bushing that carries a saline-filled guide tube made of fused silica (Plastics One) for visualization during MRI sessions. The small internal diameter $(0.15 \mathrm{~mm})$ of the fused silica probe facilitates accurate localization. Our recording tracks vertically penetrated the anterior bank of the superior temporal sulcus and entered area MSTd (Fig. 1). All recording sites in all three monkeys were in dorsal MST.

Visual stimuli and task. Monkeys were comfortably seated with the head stabilized in the horizontal stereotaxic plane. They were rewarded with fruit juice every $0.5-1 \mathrm{~s}$ for maintaining fixation on a red spot presented on the screen. Visual stimuli were rear projected onto a tangent screen placed $61 \mathrm{~cm}$ from the eyes, covering a maximum visual angle of $77 \times 77^{\circ}$. Stimuli were projected using a Mirage 2000 Digital Light Projector (Christie Digital) with resolution $1024 \times 1024$ pixels, frame rate 96 $\mathrm{Hz}$ and mean luminance $170 \mathrm{~cd} / \mathrm{m}^{2}$ (Price et al., 2005). The Mirage 2000 does not suffer from typical refresh and scanning problems associated with CRT or LCD displays. Images are projected by light reflected from an array of micro mirrors, with each pixel's color controlled by blending red, green, and blue light from three independently controlled mirrors. Each mirror can be set ON to project light to the screen or OFF to absorb the light. The luminance of each pixel is controlled by the proportion of the time that each mirror is set to the $\mathrm{ON}$ position. Because each mirror can be moved to an ON or OFF position thousands of times per frame and each pixel in a frame is updated simultaneously, there are no problems with phosphor luminance decay or scanning and refresh flicker as seen with CRT and LCD displays.

Stimuli were random texture patterns formed of $0.8^{\circ}$ black or white squares, with contrasts of either 50 or $99 \%$. Sixty cells were tested with $50 \%$ contrast and 7 cells with $99 \%$. Results from both contrasts showed indistinguishable characteristics, so population data show all 67 cells combined. The stimulus was presented for a single frame at random times relative to saccade onset. Before the experiment, we determined the preferred direction and speed of a cell using moving texture patterns, while the animal fixated a central, stationary target. We also used a small patch of moving dots that could be placed at any location on the screen. The dots in the patch moved in the cells preferred direction. We moved the patch of dots around using a computer mouse to establish the borders of the receptive fields. For the cells presented we could locate the edge of the receptive field within the bounds of the screen borders. We cannot preclude extra-classical receptive field effects but we are confident that the primary excitatory regions of the receptive fields did not include the screen borders.

During the saccade experiment, monkeys were required to fixate the presented spot while we presented the flashed stimulus. Whenever the location of the spot changed to the alternate position the monkey was required to make a saccade to it as soon as possible. Once the eye landed on the new spot location the monkey was rewarded with fruit juice. They were then rewarded every $0.5-1 \mathrm{~s}$ for continuing to fixate the target. Flashes were presented at intervals of between 100 and $500 \mathrm{~ms}$. The experimental protocol aimed to provide sufficient saccades to obtain a minimum of six flashes in each $20 \mathrm{~ms}$ bin between -200 and $+200 \mathrm{~ms}$ relative to saccade onset. However, recording stability made it necessary in some cells to terminate trials before this was achieved. As a result, for a small number of bins in certain cells less than six flashes occurred. Bins with less than two flashes were excluded from further analysis. The monkeys fixated a stationary target for extended periods between saccades, allowing us to record control responses in which no saccades were made within $\pm 500 \mathrm{~ms}$ of the flash presentations.

Monkeys were placed in complete darkness for short periods while we encouraged them to make saccades in response to auditory cues (clicking fingers, etc.) presented behind the screen (the screen was located directly in front of the monkeys). The locations of the auditory cues were regularly changed. An experimenter watching the eye movements in the control room gave feedback to the experimenter in the dark room to ensure that the monkeys remained awake and continued to actively make saccades. Darkness was achieved by covering the lens of the projector and blacking out all other light sources. Human observers reported total darkness even after $3-5 \mathrm{~min}$ in the darkened room. The testing procedure to obtain sufficient flash responses in every time window and to get 
accurate data in the dark was very time consuming. As it was often difficult to hold cells for the entire duration of the experiments, we present data from 67 cells in which all data sets were acquired.

Data collection. Eye position in two dimensions was measured using a magnetic coil system (CNC Electronics) and sampled at $1 \mathrm{kHz}$. Single-unit activity was measured using irontipped, epoxy-coated tungsten electrodes (Frederick-Haer Corporation) with impedances ranging from 1 to $4 \mathrm{M} \Omega$. Single-unit activity was sampled at $25 \mathrm{kHz}$ and stored for offline analysis. Action potentials were detected online with an analog window discriminator (Alpha-Omega). The output from the window discriminator together with saccade target and stimulus timing signals were logged as event markers in register with the eye position and unit activity signals. All signals were digitized with 16-bit precision using a Power 1401 acquisition system (CED).

Data analysis. Eye velocity was calculated offline by differentiation of the eye position signals using a finite difference formula with temporal resolution of $1 \mathrm{~ms}$. Saccade onset was then defined as the moment when the eye velocity first exceeded $10 \%$ s. Stimulus onset was determined by way of a frame synchronous event marker generated by the stimulus computer. Spike arrival times were determined off-line through action potential template matching (Spike2; CED).

Stimulus-evoked responses were binned in $20 \mathrm{~ms}$ bins based on the stimulus onset time relative to the onset of the nearest saccade. Responses elicited by flashes delivered $>500 \mathrm{~ms}$ before or after the onset of a saccade were designated as control responses. Each neuronal response was then represented as a spike density function (SDF) with $1 \mathrm{kHz}$ resolution generated by convolution of a Gaussian kernel of unit area and $\sigma=5 \mathrm{~ms}$ with a train of Dirac delta functions, one delta function corresponding to the arrival time of each spike. Mean SDFs were then calculated by averaging responses to individual stimulus presentations within each $20 \mathrm{~ms}$ bin and for the control condition. For each cell, the spontaneous rate within each $20 \mathrm{~ms}$ time bin was estimated based on a period of $25 \mathrm{~ms}$ immediately after stimulus presentation, averaged across all trials aligned at stimulus onset.

Response latencies were calculated relative to stimulus onset using a Poisson analysis of the spike rate. The intrinsic spontaneous rate of each cell was estimated based on a period of $25 \mathrm{~ms}$ immediately after stimulus presentation, averaged across all control trials aligned at stimulus onset. No cell had $<244$ trials included in the control condition. Spike rates within each bin, and for the control condition were then compared with a Poisson distribution fitted to the intrinsic spontaneous rate to identify periods of significant modulation of the spike rate. Response latency was defined as the beginning of the first period of significant modulation during which the spike rate exceeded the $99 \%$ cutoff of the Poisson distribution fitted to the spontaneous rate for at least $25 \mathrm{~ms}$. Trial-by-trial variability in response latency was estimated using the method of Nawrot et al. (2003). In brief, for a given stimulus condition we calculated the relative latency for each trial which maximized the total pairwise cross-correlation of the single trial SDFs, subject to the constraint that the mean of the relative latencies was zero. The resulting distribution of the estimated relative latencies of the individual trials then provided a direct quantification of the variability in the response latency (which we plot as SEs).

\section{Results}

Time course of saccadic modulation

Trained monkeys made rewarded saccades back-and-forth between two alternately presented fixation points separated by $10^{\circ}$.
The fixation points were each offset horizontally by $5^{\circ}$ from the center of the stimulus screen (Fig. $2 \mathrm{~A}$, black dots). For most of the time, the only visual stimulus present was one of the red fixation targets (the stimulus screen was gray and covered the entire receptive fields of the recorded neurons). We recorded from neurons in MSTd of the parietal cortex in three monkeys. Most neurons were highly direction-selective but also gave robust responses to wide-field flashed stimuli. At random intervals relative to the saccades we presented a briefly flashed full-field, checkerboard pattern to the monkeys (Fig. $2 A$ ). Figure $2 A$ shows a mean eye trace from five separate saccades aligned at saccade onset (time 0). For each of the five separate saccades one $10 \mathrm{~ms}$ flashed stimulus was presented at a random interval either before or after saccade onset (the times of flash onset relative to saccade onset are shown by dashed lines). Below the eye trace we show the first $100 \mathrm{~ms}$ of the spike train observed after the onset of each flash. The same spike trains are shown in Figure $1 B$, aligned at flash onset to allow comparison of response timing.

The cell shown had a very low spontaneous rate and produced a single time-locked spike in response to the flashed stimulus. For the flashes presented $>100 \mathrm{~ms}$ before saccade onset, this response had a mean latency of $67 \mathrm{~ms}$. For the flash presented $65 \mathrm{~ms}$ before saccade onset there was no response (Fig. $2 A$ ). The last flash presentation shown in Figure 2 was presented $81 \mathrm{~ms}$ after saccade onset. Two changes to the response, relative to the presaccadic presentations, are clear: (1) more spikes were generated by the postsaccadic stimulus than in the presaccadic case and (2) the spikes occurred in MSTd earlier than for the presaccadic flashes (Fig. $2 B$ ). These observations were highly repeatable and typical of the cells in our population.

For quantitative analysis, flash responses for each cell were binned and averaged based on the time of stimulus delivery rel- 


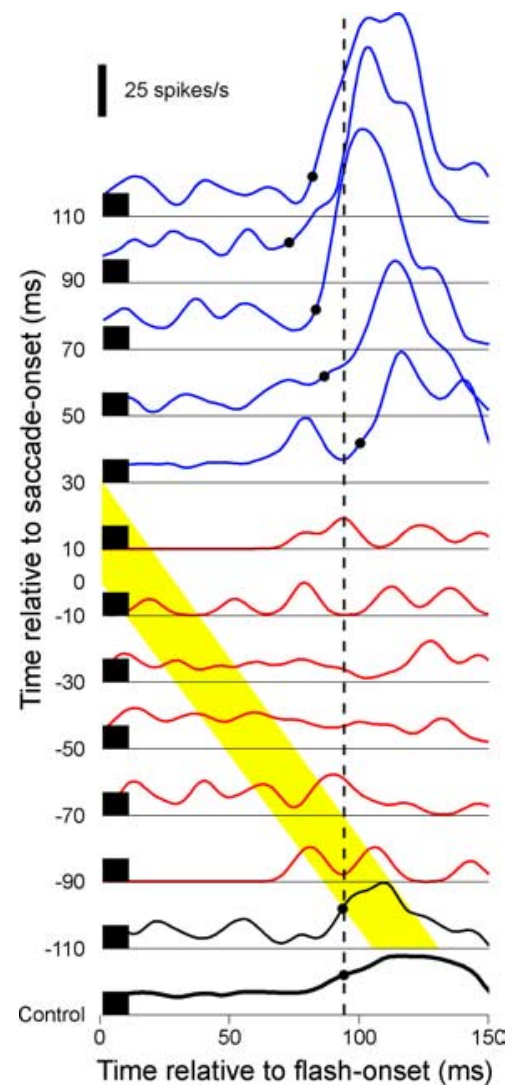

Figure 3. Spiking activity around the time of saccades. Responses of an MSTd neuron to 10 ms flashed stimulus presentations presented as SDFs aligned at flash onset. The black boxes show the duration of the flash. The lowest trace shows the control response (no saccades within $\pm 500 \mathrm{~ms}$ ). The $y$-axis shows time of stimulus presentation relative to saccade onset. Each SDF represents the mean of all responses generated by flashes within a 20 ms time window centered on the indicated time relative to saccade onset. The yellow area shows the mean saccade duration. The black dots show measured response latency (vertical dashed line indicates the latency of the control).

ative to saccade onset. The bins were $20 \mathrm{~ms}$ in duration and were centered at $20 \mathrm{~ms}$ intervals between -490 and $+490 \mathrm{~ms}$ relative to saccade onset (we only present results from -190 to +490 because response amplitudes before -190 were very stable). Spike trains elicited in response to flashes presented within each bin were aligned at the time of flash onset and the mean response expressed as a spike density function. The lowest trace in Figure 3 shows the mean response of a single cell to flashed stimuli presented without saccades (i.e., no saccades within $\pm 500 \mathrm{~ms}$ ). Above the control trace, mean responses for each bin are aligned vertically relative to flash onset (black rectangles show flash durations). The numbers on the $y$-axis show the position of the bins (i.e., the time of the flash presentations) relative to saccade onset. The yellow region shows the mean saccade duration. For all cells in MSTd there was a delay between the flash presentation and the observed response (latent period). Thus, for most cells even flashes presented before saccade onset (Fig. 3, below and to the left of the yellow region) led to responses in MSTd after saccade onset (Fig. 3, above and to the right of the yellow band).

For the cell shown in Figure 3, the control latency was $93.0 \mathrm{~ms}$ (SE, $0.7 ; n=967$ ) and the peak response occurred 100-120 ms after the flash. For stimulus flashes presented $110 \mathrm{~ms}$ before the saccade the responses in MSTd occurred at or soon after saccade onset and had similar amplitudes to the control. When flashes occurred from -70 to +20 ms relative to saccade onset responses in the usual response time window were greatly suppressed relative to controls (red traces). When flashes occurred $>20 \mathrm{~ms}$ after saccade onset the response amplitudes (blue traces) were enhanced relative to responses to presaccadic flashes. This enhancement is obvious when the blue traces are compared with the responses to the control (black). The black dots in Figure 3 show the mean latency of responses in each $20 \mathrm{~ms}$ bin. For comparison, the dashed vertical line shows the control latency. For bins where no latency is indicated it was not possible to reliably measure response latency (see Materials and Methods). It is clear that for the cell shown postsaccadic flashes led to decreased response latencies.

Figure $4 A$ shows relative mean peak-response amplitudes for a single neuron as a function of stimulus time relative to saccade onset (all response amplitudes normalized to controls). For this cell the first significant and persistent (i.e., occurring for at least three consecutive $20 \mathrm{~ms}$ bins) suppression of the visual response occurred $90 \mathrm{~ms}$ before saccade onset (leftmost red arrow). The maximum suppression occurred $10 \mathrm{~ms}$ after saccade onset (second red arrow). Flashes presented at the end of a saccade (yellow zone) generated responses that were for the first time significantly larger than controls (leftmost blue arrow). This postsaccadic enhancement peaked $90 \mathrm{~ms}$ after saccade onset (second blue arrow) and persisted until $310 \mathrm{~ms}$ after saccade onset (third blue arrow), at which point the response amplitudes fell to or below the control value for three consecutive time windows. We measured the times for these five amplitude markers for all cells where possible. Figure 4, $B-F$, shows the distribution of these markers relative to saccade onset across the population. Significant suppression first occurred usually $30 \mathrm{~ms}$ before saccade onset (Fig. $3 B$ ) although 12 cells $(18 \%)$ showed significant suppression $>60$ ms before saccade onset. Maximum suppression usually occurred when flashes were very close to saccade onset (Fig. $4 C$ ), which equates exactly with perceptual observations (Diamond et al., 2000). The first significant enhancement above the control level occurred most often 50 ms after saccade onset (Fig. 4D), which equates well with the peak enhancement observed for saccades in darkness (see Fig. $6)$. The maximum enhancement occurred 100-130 ms after saccade onset (Fig. $4 E$ ). The enhancement phase terminated $\sim 200$ ms after saccade onset (Fig. $4 F$ ), which again equates well with the data from saccades in darkness (see Fig. 6). For each cell we measured the maximum suppression and enhancement relative to control amplitudes. The mean reduction in response amplitude compared with controls at the point of maximum suppression was $81 \pm 8 \%($ mean $\pm \mathrm{SE}, n=67)$. The mean peak enhancement was $310 \pm 62 \%$ (mean $\pm \mathrm{SE}, n=67$ ) of the control value. As is evident from the large SE, the amount of enhancement was quite variable between cells. Some cells produced almost no control response but very large visual responses in the wake of saccades (sometimes more than a tenfold increase). Conversely, a number of cells showed very moderate enhancement, with two cells showing no significant enhancement at all.

\section{Response latency}

We have shown that response amplitudes are suppressed for flashes presented before and during saccades and enhanced for flashes presented after saccades. We also found that the latency of these neurons changed around the time of saccades. In most cells response latencies for flashes presented after saccade-end were significantly lower than control values. This is illustrated in Figure $5 A$, which shows the control response of a cell (black trace) and the response to flashes presented $50 \mathrm{~ms}$ after saccade onset (blue trace). The response to the postsaccadic flash is larger than 
the response under the control condition. It is also clear that the postsaccadic response exceeds the Poisson threshold used to determine response latency significantly earlier than in the control condition. For the example shown, the control latency was $42.7 \pm 3.2 \mathrm{~ms}$, whereas the postsaccadic latency was $35.1 \pm 2.2 \mathrm{~ms}$ (mean \pm $\mathrm{SE}$ ). The $7.6 \mathrm{~ms}$ reduction in latency was highly significant ( $t$ test, $p<0.05$ ).

For each cell in our population, we determined the response latency for flashes delivered in each $20 \mathrm{~ms}$ bin relative to saccade onset and compared them with the cell's control latency. Figure $5 B$ shows an example in which we compare the response latencies for 45 cells under control conditions with those for flashes presented $50 \mathrm{~ms}$ after saccade onset. The dashed line indicates the line of equality of the two measures. Most cells exhibit a significant decrease in response latency for flashes presented $50 \mathrm{~ms}$ after saccade onset. The distribution of this change in response latency for all 45 cells is shown as a histogram in Figure 5C. For the cells shown, there was a significant decrease in latency of $-16.8 \pm 2.2 \mathrm{~ms}$ (mean $\pm \mathrm{SE}, n=45, t$ test, $p \ll 0.001)$. In making this comparison we were able to calculate latency differences for only 45 of the 67 cells in our population. The reason for the reduced cell count was that for this particular bin 22 cells had either too few flash responses (see Materials and Methods) or the flash response amplitude was small relative to the spontaneous rate. In 14 of these cells, flash responses under the control condition did not cross the Poisson threshold, making it impossible to get a reliable latency measure. Figure $5 D$ shows the change in response latency in each $20 \mathrm{~ms}$ bin as a function of stimulus delivery time (i.e., bin position) relative to saccade onset. The mean latency data presented for each bin represents the average latency from between 26 and 46 cells. The overwhelmingly significant finding was that response latencies for flashes presented between 30 and $250 \mathrm{~ms}$ after saccade onset were significantly reduced (Fig. 5D).

It can be argued that the reduction in response latencies illustrated in Figure 5 may be an iceberg effect. That is, the response waveform remains unchanged but is shifted upward relative to the response threshold. We therefore repeated our analysis, determining response latencies simply as the first moment after stimulus presentation at which the mean spike rate exceeded a threshold equal to half its maximum amplitude (above the spontaneous rate) and subsequently remained in excess of that threshold for at least $10 \mathrm{~ms}$. Response latencies determined in this way were reduced for flashes delivered $50 \mathrm{~ms}$ after saccade onset by $-8.37 \pm 1.73 \mathrm{~ms}$ (mean $\pm \mathrm{SE}, n=59$ ) relative to the control condition. Although more modest than the reduction based on the Poisson analysis of the spike rate $(-16.8)$, this reduction is nevertheless significant at the population level (one-tailed $t$ test, $p<0.015)$. The smaller mean latency reduction and the concomitant reduction in statistical significance is to be expected, because using a "half-height" threshold has a normalizing effect on the mean spike rate functions - in effect nullifying any postsaccadic enhancement of excitability. In light of this, the fact that the reduction in latency remains significant at the population level is instructive.

\section{Origins of saccadic modulation}

Up to this point we have described the effects of saccades on stimulus-driven spiking activity. However, we also observed that in addition to the modulations of the flash responses described so far, the mean spontaneous activity of our cells was often also increased after saccades. To quantify this nonvisual effect we calculated the prevailing spontaneous rate within each $20 \mathrm{~ms}$ time bin. Figure $6 \mathrm{~A}$ shows the modulation in spontaneous spiking activity relative to the control condition around the time of saccade onset for our cell population $(n=67)$. Spontaneous activity increased significantly above the control spontaneous rate before saccade onset $(-10 \mathrm{~ms})$ and persisted until $\sim 120 \mathrm{~ms}$ after saccade onset (Fig. $6 \mathrm{~A}$, asterisks). However, in contrast to the modulation of visual responses described above, there was no significant suppression of spontaneous spiking activity at any time relative to the saccades. It can be argued that identifying suppression of spontaneous activity is difficult from a population in which some cells have zero spontaneous activity (Fig. 2, cell). As the data in Figure $6 A$ is normalized we checked the absolute spontaneous rates in the period from -200 to $-100 \mathrm{~ms}$ before saccade onset and compared these with the mean activity level in the $100 \mathrm{~ms}$ preceding saccade onset. The mean spontaneous rate for the population was $13.2 \pm 10.7$ spikes/s. The mean spiking rate in the $100 \mathrm{~ms}$ before saccade onset was $13.1 \pm 10.7$ spikes/s. These two values are not significantly different ( $t$ test, $p=0.79$ ), showing that in the time window associated with suppression in 

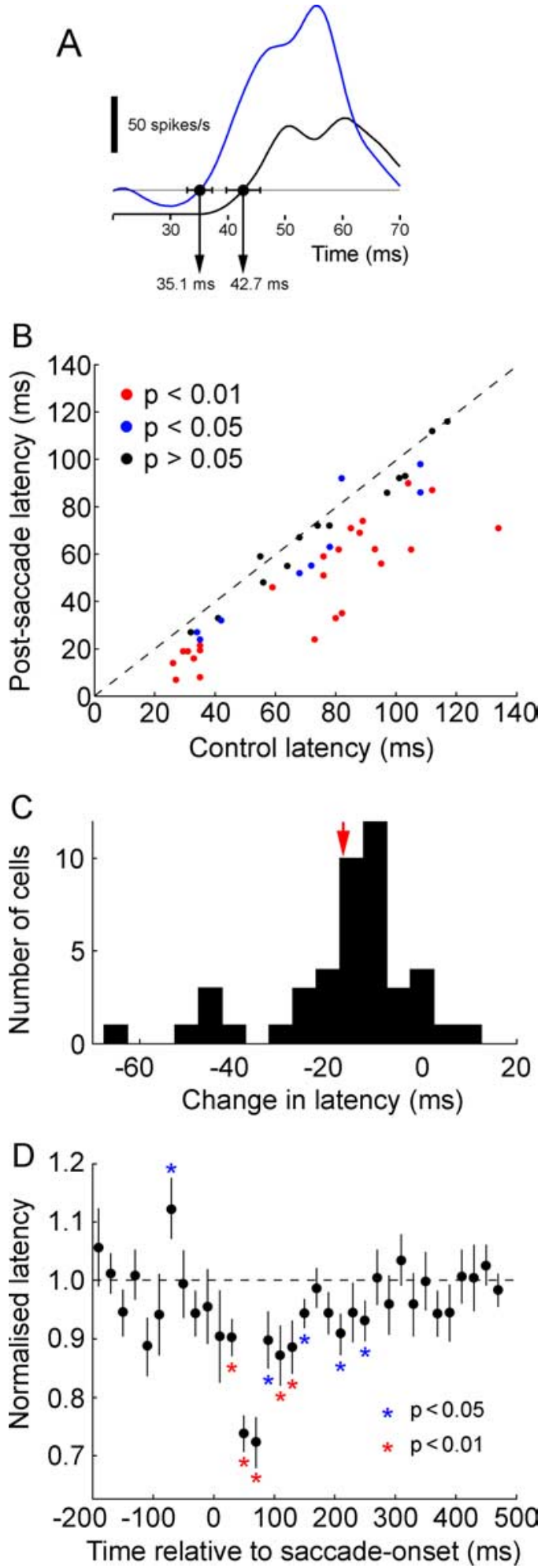

Figure 5. Latency changes after saccades. $A$, Mean responses generated by stimulus flashes presented under control conditions (black) and $50 \mathrm{~ms}$ after (blue) saccade onset. The horizontal line shows the threshold, defined as the $99 \%$ cutoff of the Poisson distribution fitted to the spontaneous rate, used to determine response latency. Horizontal error bars indicate SES. The reduction in latency for this cell was $7.6 \mathrm{~ms}$. $\boldsymbol{B}$, Cell-by-cell comparison of neuronal response latencies for visual stimuli delivered under control conditions (no saccades) and for stimuli delivered $50 \mathrm{~ms}$ after saccade onset. The dashed line shows the line of equality for the two measures. Black dots: not significantly different; blue dots: significantly different ( $t$ test, $p<$ $0.05)$; red dots: significantly different $(t$ test, $p<0.01)$. C, Histogram showing the distribution of cells based on their latency reduction. The red arrow indicates the mean latency reduction $(-16.78 \pm 2.22 \mathrm{~ms}$, mean $\pm \mathrm{SE}, n=45)$ for the population. $\boldsymbol{D}$, Normalized response latency
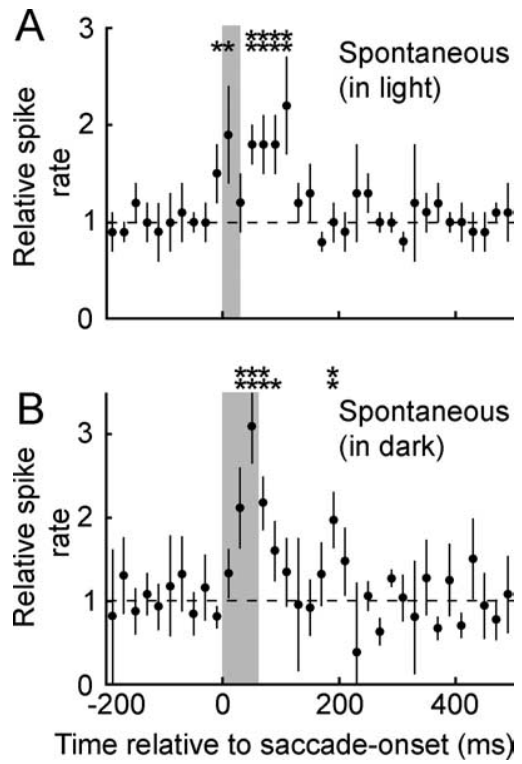

Figure 6. Origins of saccadic modulation. $\boldsymbol{A}$, Mean spontaneous activity in each $20 \mathrm{~ms}$ bin plotted as a function of time relative to the onset of saccades performed while viewing a bright, blank screen. Spontaneous rates for each cell were normalized to the control condition (no saccades). Gray band shows saccade duration. $\boldsymbol{B}$, Mean spontaneous activity as a function of time relative to the onset of saccades in total darkness. Again, spontaneous rates were normalized to the control condition for each cell. The gray band shows the maximum saccade duration (saccades varied from 15 to $62 \mathrm{~ms}$ ). In $\boldsymbol{A}$ and $\boldsymbol{B}$, asterisks indicate significant enhancement of spontaneous activity relative to the control condition $\left({ }^{*} p<0.05,{ }^{* *} p<0.01, t\right.$ test). Error bars indicate SEs for the cell population ( $n=67)$.

the flash tests we see no reduction in spontaneous rate during saccades in darkness. Importantly, on average the cells have sufficient spontaneous activity to reveal suppression if it was present. Together, the results above suggest that MSTd is not itself suppressed around the time of saccades; rather, suppression of visual responses is inherited from earlier visual areas.

Given that the increase in spiking activity occurs primarily after the saccade it is conceivable that the increase might be a response to visual stimulation during the eye movement. For example, the fixation spot or the peripheral screen border could provide some visual stimulation (see Materials and Methods). We discount these possibilities for two reasons. First, preliminary tests showed that the cells were highly direction-selective but they showed the same postsaccadic increase in activity for saccades in all tested directions (ANOVA, $p>0.05$, data not shown). Second, in most cells, and therefore in the population average (Fig. $6 \mathrm{~A}$ ), a significant increase in activity occurred before saccade onset $(t$ test, $p<0.05$ ), suggesting that the early activity change did not arise from visual input.

The evidence suggests that the increase in activity in MSTd after saccades arises from an extra-retinal signal. To test this hypothesis further we looked for significant changes in spontaneous activity during saccades in complete darkness. Monkeys made saccades in response to auditory cues presented at various locations in front of the animals. That is, an experimenter stood

$\leftarrow$

as a function of stimulus delivery time relative to saccade onset for the cell population ( $n=$ $26-46$, not all cells were included in every point, see Materials and Methods). It is apparent that significant latency reductions occur for flashes delivered +30 to $+250 \mathrm{~ms}$ after saccade onset. Asterisks indicate whether the reduction in population mean compared with controls is significant (color code as in $\boldsymbol{A}$ ). Error bars indicate SEs. 


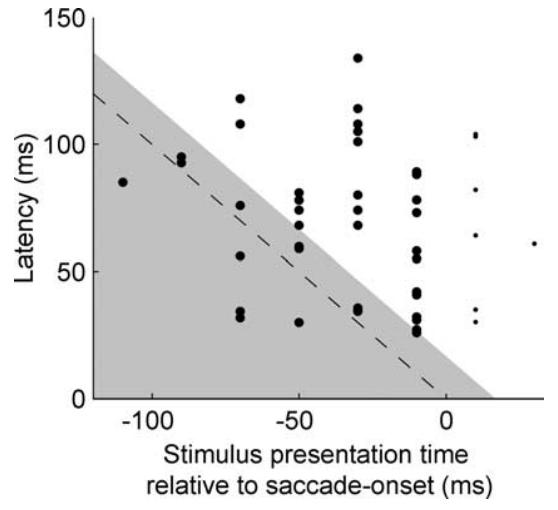

Figure 7. Response latency versus time of suppression (relative to saccade onset). Cell-bycell comparison of control latency and the time of first suppression, defined as the earliest time of stimulus delivery relative to saccade onset for which the observed response was significantly suppressed relative to the control condition $(n=46)$. Note that some points are plotted on top of each other. The dashed diagonal line denotes the time of saccade onset. Seven cells showed significant suppression only for stimuli delivered after saccade onset (small filled circles). The remainder of the cells showed significant suppression of visual responses for stimuli delivered before saccade onset (large circles). Of these, 14 cells showed significant suppression of responses expected to arrive in MSTd within $16 \mathrm{~ms}$ after saccade onset (shown in gray shaded area). The gray areas in $\boldsymbol{A}$ and $\boldsymbol{B}$ show those cells in which the suppression could not have arisen from retinal mechanisms.

behind the viewing screen and made noises to attract the monkey's attention to various locations, and therefore make saccades, in the dark. Analysis was confined to saccades with amplitudes of $5-20^{\circ}$. There was no significant evidence of suppression of spontaneous activity during saccades (Fig. 6B). However we did observe a significant increase in spiking activity that peaked $50 \mathrm{~ms}$ after saccade onset, regardless of saccade duration. This increased activity generally lasted no longer than $200 \mathrm{~ms}$. As this modulation of spike-rate occurred in complete darkness, we presume that it arose from an extra-retinal signal.

The idea that suppression arises from an extra-retinal signal is further supported by analysis of the relationship between the intrinsic latency of each cell and the time course of the suppression. For example, using a hypothetical cell with an $80 \mathrm{~ms}$ intrinsic latency, a stimulus presented $90 \mathrm{~ms}$ before saccade onset would lead to a response $10 \mathrm{~ms}$ before saccade onset. If the response was significantly attenuated relative to controls we would conclude that the suppression had an extra-retinal origin and was initiated before the eye movement. Conversely, if a flash was presented to the same cell $10 \mathrm{~ms}$ before saccade onset we would expect a response $70 \mathrm{~ms}$ after saccade onset. If this response was attenuated we would not be able to exclude the possibility of the suppression being of retinal origin. This is because suppression could have originated in the retina and been sent into the visual system by a shorter latency route, to intercept the signal carrying information about the flash response to MSTd.

The $y$-axis in Figure 7 shows the control latencies for visual responses of 46 cells. The $x$-axis shows the time of stimulus presentation, relative to saccade onset, that first led to a significantly suppressed response. Of the 46 cells shown, seven cells exhibited suppression only for stimuli delivered after saccade onset (Fig. 7, small dots), whereas the majority showed suppression for stimuli delivered before saccade onset (large filled circles to the left of zero on the $x$-axis). For five of the latter group, the suppressed response actually arrived in MSTd before saccade onset (below the dashed diagonal line). The five points below the line cannot be accounted for simply through random variation because in order for the time of first suppression to be counted the spiking activity needed to fall below the spontaneous rate for at least 60 ms ( 3 consecutive time windows). Also, we used a $t$ test to compare the mean latency of the cell with the time of saccade onset. For all five cells the signals arrived significantly before saccade onset ( $p \ll 0.01$ ). These five cells were unequivocally suppressed by a nonretinal mechanism. We believe that in fact all cells in the gray shaded area in Figure 7 are almost certainly suppressed by an extra-retinal mechanism, and this is outlined in detail (see Discussion, Saccadic suppression).

\section{Discussion}

\section{Saccadic suppression}

Because the activity of MSTd neurons directly affect visual perception (Celebrini and Newsome, 1995) and all cells exhibited saccade-induced response modulations, it is likely that perception is altered by the effects we describe. Visual responses of neurons in MSTd were suppressed when the stimulus appeared from -90 to +50 ms relative to saccade onset. This time scale matches the time course of suppressed cortical evoked potentials and perceptual suppression of contrast sensitivity in humans (Duffy and Lombroso, 1968; Diamond et al., 2000).

What is the origin of the suppressive effect? One theory suggests that extra-retinal signals arise from the eye movement generating regions of the brain (Ross et al., 2001). Recent evidence supports a nonretinal origin for saccadic-suppression of neural activity in the pigeon visual system (Yang et al., 2008). In primates, if the LGN was suppressed before saccade onset by signals sent at the same time as those initiating the saccade, there would be presaccadic suppression (Thilo et al., 2004). These suppressed signals would then propagate through the visual pathways and be observed as reduced visually evoked activity in MSTd. LGN neurons are suppressed during saccades in the dark (Lee and Malpeli, 1998; Royal et al., 2006). However, without visual stimulation we observed no saccadic suppression in MSTd. Thus, we exclude any direct extra-retinal suppression of MSTd and posit that saccade related suppression in MSTd is inherited from previous visual areas.

A second theory explaining suppression suggests that it arises from activity changes in the retina generated by the rapid eye movements during saccades. The argument suggests that rapid acceleration at saccade onset tilts the photoreceptors, which generates a transient reduction in luminance sensitivity (Richards, 1969; Castet et al., 2001). If luminance is briefly reduced in fixating humans there is a reduction in visual sensitivity, maximal 20-50 ms after the decrement and a relatively weak reduction in visual sensitivity up to $25 \mathrm{~ms}$ before the decrement (Poot et al., 1997). Thus, a simple reduction in stimulus brightness can cause a reduction in visual sensitivity that can be perceived to occur before the stimulus was even presented. It has also been suggested that the observed reversal in directional tuning of some MST neurons during saccades might cancel any perceived motion (Thiele et al., 2002). Both suggestions are plausible explanations for stimuli presented after saccade onset. However, assuming that there is no training that allows stimulus prediction, there is no way for the visual system to alter its sensitivity before stimulus onset. Thus, any explanation for prestimulus events must come from errors in perceived timing, which incorrectly attributes visual responses with prestimulus time epochs. Examples of this postdiction are widespread in the perceptual literature (Poot et al., 1997; Krekelberg et al., 2003; Ostendorf et al., 2006) and are thought to be caused by variable visual latencies between cells and long temporal integration times.

What sets our data apart from perceptual studies is that we 
directly recorded the neural responses of single cortical neurons. Thus, our results are unencumbered by the vagaries of perception. Moreover, unlike previous physiological studies we recorded activity generated by stimuli presented before saccade onset. In our MSTd cell population 15\% of cells showed suppression for the first time only when flashes were presented after saccade onset (Fig. 7, small dots). For these cells it is impossible to decide between a retinal or extra-retinal origin for suppression. Eleven percent ( 5 of 46 ) of neurons unequivocally demonstrated extra-retinal suppression that was initiated before saccade onset (Fig. 7, below the dashed line). The remaining 74\% (34 of 46) of cells fell into a middle group. However, a simple logical argument suggests that many of the middle group were suppressed by an extra-retinal mechanism. The shortest reported response latencies for neurons in the LGN, the first postretinal stage of the retino-cortical pathway is $16 \mathrm{~ms}$ (Maunsell et al., 1999). As an example, let us take the case where a flash occurred $30 \mathrm{~ms}$ before saccade onset and the MSTd neuron had a latency of $40 \mathrm{~ms}$. The visual response passed through all the stages of the visual pathways and arrived in MSTd $10 \mathrm{~ms}$ after saccade onset, and was suppressed relative to controls. For the suppression to have been of retinal origin a signal at saccade onset would need to have intercepted the previously initiated flash response before it arrived in MSTd. Given that no known signal can reach even the LGN in $<16 \mathrm{~ms}$ there is no way that the suppressing signal could possibly have intercepted the flash response as it had already arrived in MSTd. Based on this type of logic, at least 14 cells (30\% of the total cell count) showed saccadic suppression of nonretinal origin (Fig. 7, shaded area). We cannot conclude that all of the reduced visual sensitivity observed during saccades is of extraretinal origin, but we are confident that a presaccadic effect arises from a centrally generated suppression of early visual processing.

\section{Postsaccadic enhancement}

There is a long postsaccadic period (up to $450 \mathrm{~ms}$ ) in which spontaneous activity increases, and stimulus presentation generates enhanced visual responses. Performance in a number of visual tasks is enhanced after saccades: e.g., contrast sensitivity when viewing isoluminant color modulated stimuli is maximally enhanced 50-100 ms after saccade onset (Burr et al., 1994). Interestingly, evidence for perceptual postsaccadic enhancement is weaker when viewing luminance modulated stimuli, as were used in our experiments (Burr et al., 1994). This dependence on stimulus modality may prove central in reconcile neuronal observations with psychophysical studies.

Spiking rates in the LGN during spontaneous saccades made in darkness exhibit both presaccadic suppression and postsaccadic enhancement (Ramcharan et al., 2001; Reppas et al., 2002; Royal et al., 2006). It therefore seems reasonable that both suppression and enhancement of activity in MSTd may be inherited from the LGN via intermediate visual areas. Although we observed strong enhancement of spiking activity in MSTd after saccades (even in darkness), we observed no significant suppression of spontaneous activity for saccades in darkness. Thus, there appears to be a direct enhancement of MSTd during the postsaccadic period but no direct suppressive mechanism. We presume that for visual stimuli both suppression and enhancement are inherited from earlier visual areas. Once the signal arrives in MSTd no additional suppression is imposed but enhanced signals may well be further boosted.

That visual responsiveness is enhanced after saccades indicates that saccades are critically related to visual sensitivity: without saccades, visual sensitivity is reduced (Ibbotson et al., 2007;
Rajkai et al., 2008). Thus, saccades not only provide a means to shift gaze direction but also to increase visual sensitivity when saccades are frequent. We conclude that the sensitivity of the visual system depends on activity in the motor system. Our data supports a model in which the visual and motor systems have not developed independently, but rather have evolved to optimize their mutual performance.

\section{Time compression}

How could our results relate to the saccade-related changes in perceived time that have been observed in human subjects? A significant difference in response latencies to visual stimuli presented before and after saccades could explain time compression (Ibbotson et al., 2006). Briefly, if one flash is presented before the saccade and a second flash during the postsaccadic period the interresponse interval will be consistently shorter than the interstimulus interval if the latency of the second response is shorter than that of the first. As a result, there will be a physical compression of the interresponse interval, presumably leading to a compression of perceived time. For a restricted range of interstimulus intervals and presentation times relative to saccade onset, it has been shown that the perception of time can be reversed (Morrone et al., 2005): the stimulus presented first is perceived as occurring second. The power of the theory just outlined is that it can account for this temporal inversion as well as time compression. Recently, another theory to explain time compression was proposed (Terao et al., 2008). It suggests that weak transient responses to flashed stimuli, as occurred in our study, might fail to trigger the detection of temporal asynchrony between consecutively flashed stimuli. The resulting bias toward simultaneity may lead to an apparent time compression. However, this theory cannot account for perceived temporal inversion.

Our physiological data show that response latencies for flashes presented after saccades are reduced. Psychophysical measures of impulse responses to flashed stimuli in humans also suggest that responses during saccades are attenuated and have an earlier time-to-peak (12 ms vs $20 \mathrm{~ms}$ without a saccade) (Burr and Morrone, 1996). Thus, there will be a physical reduction in the interresponse interval for two flashes presented either side of a saccade. Our findings provide strong support for a theory of time compression that depends on differential latencies before and after saccades (Ibbotson et al., 2006). However, in attempting to reconcile our physiological findings with human psychophysical studies there is a problem. For stimuli delivered $50 \mathrm{~ms}$ after saccade onset the latencies of MSTd neurons are reduced on average by $\sim 17$ ms relative to controls. However, perceptual time reductions can be as large as $50 \mathrm{~ms}$ in human observers (Morrone et al., 2005). We propose incorporating the theory of Terao et al. (2008) into our theory based on differential response latencies. Our physiological data provide evidence that responses before saccades are suppressed and that postsaccadic responses have reduced latencies. Thus, the data supports both theories of time compression. First there will be ambiguity in detecting the asynchrony of consecutive flashes attributable to saccadic suppression. Second, if signals are strong enough to provide some measure of interflash interval, the interval will be underestimated because of reduced postsaccadic latencies. If both effects work simultaneously the overall perceptual compression will be larger than expected from either effect alone. The elegance of combining the theories is that time compression of a scale observed perceptually can be explained, together with temporal inversion where stimulus conditions are appropriate. 


\section{References}

Burr DC, Morrone MC (1996) Temporal impulse response functions for luminance and colour during saccades. Vision Res 36:2069-2078.

Burr DC, Holt J, Johnstone JR, Ross J (1982) Selective depression of motion sensitivity during saccades. J Physiol 333:1-15.

Burr DC, Morrone MC, Ross J (1994) Selective suppression of the Magnocellular visual pathway during saccadic eye-movements. Nature 371:511-513.

Castet E, Jeanjean S, Masson GS (2001) 'Saccadic suppression' - no need for an active extra-retinal mechanism. Trends Neurosci 24:316-318.

Castet E, Jeanjean S, Masson GS (2002) Motion perception of saccadeinduced retinal translation. Proc Natl Acad Sci U S A 99:15159-15163.

Celebrini S, Newsome WT (1995) Microstimulation of extrastriate area MST influences performance on a direction discrimination task. J Neurophysiol 73:437-448.

Diamond MR, Ross J, Morrone MC (2000) Extraretinal control of saccadic suppression. J Neurosci 20:3449-3455.

Duffy FH, Lombroso CT (1968) Electrophysiological evidence for visual suppression prior to the onset of a voluntary saccadic eye movement. Nature 218:1074-1075.

Findlay JM, Gilchrist ID (2003) Active vision: The psychology of looking and seeing, pp 220. Oxford: Oxford UP.

Holt EB (1903) Eye movements and central anaesthesia. Psychol Rev $4: 3-45$.

Ibbotson MR, Crowder NA, Price NSC (2006) Neural basis of time changes during saccades. Curr Biol 16:R834-R836.

Ibbotson MR, Price NS, Crowder NA, Ono S, Mustari MJ (2007) Enhanced motion sensitivity follows saccadic suppression in the superior temporal sulcus of the macaque cortex. Cereb Cortex 17:1129-1138.

Krekelberg B, Kubischik M, Hoffmann KP, Bremmer F (2003) Neural correlates of visual localization and perisaccadic mislocalization. Neuron 37:537-545

Lee D, Malpeli JG (1998) Effects of saccades on the activity of neurons in the cat lateral geniculate nucleus. J Neurophysiol 79:922-936.

Maunsell JH, Ghose GM, Assad JA, McAdams CJ, Boudreau CE, Noerager BD (1999) Visual response latencies of magnocellular and parvocellular LGN neurons in macaque monkeys. Vis Neurosci 16:1-14.

Morrone MC, Ross J, Burr D (2005) Saccadic eye movements cause compression of time as well as space. Nat Neurosci 8:950-954.

Nawrot MP, Aertsen A, Rotter S (2003) Elimination of response latency variability in neuronal spike trains. Biol Cybern 88:321-334.
Ostendorf F, Fischer C, Gaymard B, Ploner CJ (2006) Perisaccadic mislocalization without saccadic eye movements. Neuroscience 137:737-745.

Poot L, Snippe HP, van Hateren JH (1997) Dynamics of adaptation at high luminances: Adaptation is faster after luminance decrements than after luminance increments. J Opt Soc Am A Opt Image Sci Vis 14:2499-2508.

Price NS, Ibbotson MR, Ono S, Mustari MJ (2005) Rapid processing of retinal slip during saccades in macaque area MT. J Neurophysiol 94:235-246.

Rajkai C, Lakatos P, Chen CM, Pincze Z, Karmos G, Schroeder CE (2008) Transient cortical excitation at the onset of visual fixation. Cereb Cortex 18:200-209.

Ramcharan EJ, Gnadt JW, Sherman SM (2001) The effects of saccadic eye movements on the activity of geniculate relay neurons in the monkey. Vis Neurosci 18:253-258.

Reppas JB, Usrey WM, Reid RC (2002) Saccadic eye movements modulate visual responses in the lateral geniculate nucleus. Neuron 35:961-974.

Richards W (1969) Saccadic suppression. J Opt Soc Am 59:617-623.

Rodieck RW (1988) The primate retina. In: Comparative primate biology, Vol 4: Neurosciences (Steklis HD, Erwin J, eds), pp 202-278. New York: Alan R. Liss.

Ross J, Morrone MC, Goldberg ME, Burr DC (2001) Changes in visual perception at the time of saccades. Trends Neurosci 24:113-121.

Royal DW, Sáry G, Schall JD, Casagrande VA (2006) Correlates of motor planning and postsaccadic fixation in the macaque monkey lateral geniculate nucleus. Exp Brain Res 168:62-75.

Sommer MA, Wurtz RH (2002) A pathway in primate brain for internal monitoring of movements. Science 296:1480-1482.

Sommer MA, Wurtz RH (2006) Influence of the thalamus on spatial visual processing in frontal cortex. Nature 444:374-377.

Terao M, Watanabe J, Yagi A, Nishida S (2008) Reduction of stimulus visibility compresses apparent time intervals. Nat Neurosci 11:541-542.

Thiele A, Henning P, Kubischik M, Hoffmann KP (2002) Neural mechanisms of saccadic suppression. Science 295:2460-2462.

Thilo KV, Santoro L, Walsh V, Blakemore C (2004) The site of saccadic suppression. Nat Neurosci 7:13-14.

Walls GL (1963) The vertebrate eye and its adaptive radiation. New York: Hafner.

Yang Y, Cao P, Yang Y, Wang SR (2008) Corollary discharge circuits for saccadic modulation of the pigeon visual system. Nat Neurosci 11:595602 . 